\title{
Spinal cord stimulation significantly decreases the need for acute hospital admission for chest pain in patients with refractory angina pectoris
}

\author{
S Murray, K G S Carson, P D Ewings, P D Collins, M A James
}

\begin{abstract}
Objective-To assess the impact of spinal cord stimulation (SCS) on the need for acute admissions for chest pain in patients with refractory angina pectoris.

Design-Retrospective analysis of case records.

Patients-19 consecutive patients implanted for SCS between 1987 and 1997. All had three vessel coronary disease, and all were in New York Heart Association functional group III/IV.

Methods-Admission rates were calculated for three separate periods: (1) from initial presentation up until last revascularisation; (2) from last revascularisation until SCS implantation; (3) from SCS implantation until the study date. Postrevascularisation rates were then compared with post-SCS rates, without including admissions before revascularisation, as this would bias against revascularisation procedures.

Results-Annual admission rate after revascularisation was $0.97 /$ patient/year, compared with 0.27 after SCS $(p=0.02)$. Mean time in hospital/patient/year after revascularisation was 8.3 days $v 2.5$ days after SCS $(p=0.04)$. No unexplained new ECG changes were observed during follow up and patients presented with unstable angina and acute myocardial infarction in the usual way.
\end{abstract}

Conclusions-SCS is effective in preventing hospital admissions in patients with refractory angina, without masking serious ischaemic symptoms or leading to silent infarction.

(Heart 1999;82:89-92)

Keywords: spinal cord stimulation; refractory angina pectoris; admission rate; cost effectiveness

Spinal cord stimulation (SCS) is an effective treatment for chronic angina pectoris refractory to both medical and surgical management. ${ }^{1-6}$ Many investigators have described a primary anti-ischaemic effect, assessed by exercise tests, ${ }^{2-5}$ ambulatory ECG recordings, ${ }^{56}$ stress echocardiography, ${ }^{7}$ and right atrial pacing studies with coronary sinus lactate assays. ${ }^{8}$ It is claimed that it is this antiischaemic action which accounts for the improvement in symptoms. Anderson et al published data in 1995 showing a reduction in both the number and duration of admissions after SCS implantation'; however, patients were separated into those who responded well to the treatment and those in whom there was minimal response. The groups were then analysed separately and admission rates were not directly compared. In this study we specifically examined the number of admissions and the length of stay of patients before and after SCS implantation. Acceptance of SCS has been limited by concern that the procedure may be harmful, though there is evidence that SCS does not mask acute myocardial infarction. ${ }^{10}$ An additional aim of our study was therefore to examine this claim, as well as documenting the causes of acute admission after SCS implantation.

\section{Methods}

We examined the case records of 19 consecutive patients implanted with SCS units at Taunton and Somerset Hospital between 1987 and 1997. All the patients had triple vessel coronary disease diagnosed at angiography, and experienced angina in New York Heart Association (NYHA) functional class III or IV. All the patients were considered unsuitable for further revascularisation. Fifteen of them had undergone previous revascularisation procedures (13 coronary artery bypass grafting (CABG), two percutaneous transluminal coronary angioplasty (PTCA)), and four were deemed unsuitable for any type of procedure. In the last, the starting point for assessing admissions before SCS was when the decision was made that they were unsuitable for a revascularisation procedure; from that time on, they were analysed in the "post-revascularisation" group.

All admissions for each patient were analysed, but only those caused by chest pain or suspected ischaemic heart disease were considered in the analysis. The admission notes, ECG records, and blood tests were reviewed to ensure that a correct diagnosis was reached in each case. If it was suspected that the origin of the pain was not cardiac, but no other clear aetiology was present, the admission was still counted and analysed-thus in case of doubt the pain was considered to be ischaemic. Admissions for other proven causes of chest pain (for example pleuritic pain or oesophagitis) were not included in the study. Admissions for cardiac investigations (coronary angiography) and revascularisation procedures (CABG and PTCA) were not included, nor were the admissions for SCS implantation itself.

The length (in days) of each admission was calculated. To ensure accuracy, this was 
Table 1 Patient characteristics and drug treatment before implantation

\begin{tabular}{lr}
\hline Total & 19 \\
Men & 14 \\
Women & 5 \\
Previous MI & 12 \\
$\quad$ Non-Q wave MI & 10 \\
Diabetes mellitus & 6 \\
Previous CABG & 13 \\
Previous PTCA & 2 \\
Triple vessel disease, with symptoms in NYHA class III/IV & 19 \\
Drug treatment & \\
Oral nitrates & 19 \\
Calcium channel blockers & 19 \\
B Blockers & 11 \\
Potassium channel openers & 13 \\
All four of the above & 8
\end{tabular}

CABG, coronary artery bypass graft; $\mathrm{MI}$, myocardial infarction, NYHA, New York Heart Association functional class; PTCA percutaneous transluminal coronary angioplasty.

checked against the patient database on the hospital's computer system, and any discrepancies were investigated. We were therefore confident in ensuring that all admissions had been accounted for, and that the length of stay and diagnoses were accurate. Patients were followed up at least six monthly after implantation, with ECG records at each visit. New changes on ECG were looked for and assessed.

CALCULATION OF YEARLY ADMISSION RATES

Owing to the progressive nature of coronary disease, the data were considerably skewed, with the number of admissions increasing in frequency over time. The time period between each intervention was variable and was therefore an unreliable baseline for comparison. In order to overcome this, hospital admission rates were considered per year in three periods:

- The first period was the time from first presentation up until the most recent CABG or PTCA.

- The second phase extended from the final CABG or PTCA up until SCS implantation (named the post-revascularisation period).

- The third period was from SCS implantation up to the study date (31 December 1997).

The admission rate after revascularisation was then compared with the admission rate after SCS.

STATISTICAL ANALYSIS

As the data were not normally distributed and were related to two measurements in one individual, the Wilcoxon matched pair test was performed, with a $\mathrm{p}$ value of $<0.05$ being regarded as statistically significant.

\section{Results}

The baseline characteristics of the patient group are shown in table 1 . All patients were taking maximum tolerated doses of standard antianginal drugs in combination; after implantation this treatment was continued unaltered. The mean follow up period was 2.78 years $(0.8$ to 9.0$)$. There have been two deaths within the group; the first was caused by coincidental lung carcinoma and the second by progressive heart failure in a patient with severe aortic stenosis who refused surgery.
Table 2 Admission rates before and after interventions

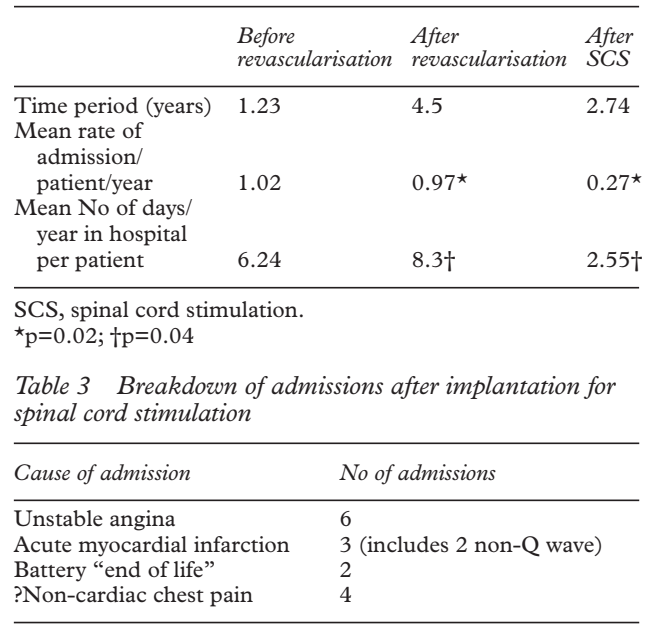

The hospital admission rates for each period are shown in table 2. Implantation of SCS led to a fall in admissions, with a decrease in the rate of admissions per patient from $0.97 /$ year to $0.27 /$ year $(p=0.02)$.

The duration of admissions following SCS implantation (table 2) also fell, the mean duration per patient of 8.3 days/year falling to 2.6 days/year $(\mathrm{p}=0.04)$.

Twelve patients did not require admission at all after SCS, the remainder accounting for all the post-SCS admissions. The admissions in the post-SCS implant period broken down by cause are shown in table 3. Most admissions were for unstable angina and acute myocardial infarction. Two patients had admissions that were thought to be non-cardiac by the time of discharge. In two other cases the devices were found to have expired batteries ("end of life"). There were three myocardial infarctions-one transmural infarct (accompanied with a ventricular fibrillation arrest) and two non-Q-wave infarcts. In all cases these patients presented with typical symptoms and were treated in the conventional manner. Follow up of patients has not revealed unexplained ECG changes, and we therefore conclude that all serious cardiac events did present to hospital.

\section{Discussion}

Several studies have shown that SCS has beneficial effects upon ischaemia. ${ }^{2-8}$ However, it was formerly unknown whether these benefits might have a significant impact on the need for hospital admission. While there is good evidence that patients feel physically and psychologically better following SCS implantation, ${ }^{11}$ it is reassuring to know that the use of these relatively expensive devices decreases hospital admissions. We did not do a formal costbenefit analysis, but table 4 gives an estimate of the costs involved. The figures are based upon the assumption that each admission consisted of 24 hours on a coronary care unit, with the remainder of the admission spent upon the general cardiology ward. From this the estimated costs per patient-year have been calculated. While these figures are not intended to give an accurate analysis, the magnitude of the difference is impressive. 
Table 4 Estimate of costs per patient-year

\begin{tabular}{lll}
\hline & Before SCS & After SCS \\
\hline 24 hours on CCU & $£ 850$ & $£ 850$ \\
Rest of admission on ward & $£ 1460(£ 200 \times 7.3$ days $)$ & $£ 320(£ 200 \times 1.6$ days $)$ \\
Total & $£ 2310$ & $£ 1170$ \\
Annual admission rate & 0.97 & 0.27 \\
Total per patient-year & $£ 2240.70$ & $£ 315.90$ \\
\hline
\end{tabular}

CCU, coronary care unit; SCS, spinal cord stimulation.

SAFETY ISSUES WITH SCS

SCS offers an effective treatment which is safe; the mortality associated with implantation is zero and the morbidity is less than $1.5 \%,{ }^{12}$ an important point when considering the other options open to those with refractory angina (such as transmyocardial laser revascularisation or cardiac transplant). Indeed Mulcahy et al (writing in 1994) felt that SCS was underused. ${ }^{13}$ The main obstacle to its wider acceptance appears to be the concern that SCS might mask angina, thereby depriving a patient of a warning signal. This concern is expressed both by doctors and by the patients themselves; it has grown from the belief that SCS works by the "gate theory" of pain. While it is true that devices such as SCS and TENS were developed in response to this theory, it was the serendipitous discovery that vascular ulcers were seen to heal when SCS was used in patients with painful peripheral vascular disease that led to the notion that SCS has specific antiischaemic effects. ${ }^{14-20}$ In the case of angina pectoris the ischaemic threshold is indeed increased-that is, ischaemia still occurs, but it does so at a higher level of myocardial work than previously. This has been elegantly demonstrated by Mannheimer et al using right atrial pacing as a stressor, and calculating lactate extraction and production as an objective measure of ischaemia. ${ }^{8}$ They showed that SCS increased the time taken for lactate extraction to become production, with the typical symptoms of ischaemia occurring at this new level. In other words, the patient still developed (and recognised) angina, but at a higher level of myocardial work. Furthermore Anderson et al showed that there was no excess of mortality in a group of 50 SCS patients when compared with data from the CASS register. ${ }^{10}$ They found that patients with acute infarcts were aware of the pain despite the SCS unit. This is reflected in our data, which show that those individuals unfortunate enough to suffer further severe ischaemia and infarction while being treated with SCS still present to hospital. We have not observed an excess mortality, and the two deaths within the group were not caused by acute coronary syndromes.

SCS is a relatively simple treatment to use, and the implantation technique is not much more invasive than permanent cardiac pacing. The most difficult part of the procedure is the insertion of the electrode, as it has to be sited in the epidural space and carefully positioned to produce stimulation in the area where angina is perceived. This technique is relatively easy in the hands of an experienced individual, such as an anaesthetist, as the basic technique is the same for insertion of epidural lines. The implantation is carried out under local anaes- thetic with sedation. Occasionally a light general anaesthetic may be used during the second stage of implantation as the "tunnelling" of the electrode from the spine to the device (usually in the axilla or subpectoral region) can be traumatic to some patients.

MECHANISM OF ACTION

There has been much speculation about the mode of action, but recent positron emission tomography (PET) studies have suggested that SCS produces a redistribution of myocardial flow from non-ischaemic to ischaemic areas. ${ }^{21}$ This has been compared with the effects of theophyllines in angina, which are also thought to act through this so called "Robin Hood" effect. $^{22}{ }^{23}$ The basis for this is thought to be adenosine antagonism, reducing adenosine mediated steal phenomena. Further evidence suggesting an adenosine blocking mechanism is the fact that SCS appeared to attenuate the effects of dipyridamole (which blocks endogenous adenosine breakdown) in the study protocol used by Hautvaust et al. ${ }^{21}$ Previously the hypothesis that SCS exerted an antisympathetic response was popular. ${ }^{12}{ }^{24}$ Mannheimer's group recently showed that SCS reduced peripheral sympathetic drive induced by right atrial pacing; the same study also examined noradrenaline spillover from the myocardium itself, but failed to show a difference. ${ }^{25}$ The technique, however, is crude and may not have been sensitive enough to detect any effect from SCS.

If SCS does act through an adenosine blocking effect, then we might also speculate that this particular group of patients possess particular characteristics that allow them to respond to this treatment. The majority of these patients not only had severe coronary disease but also extensive collateral vessels; it has been shown that these vessels are more susceptible to adenosine mediated steal syndromes than native vessels. ${ }^{26}$ It is also of interest to note that $53 \%$ of these patients have had non-Q wave myocardial infarcts previously (perhaps a disproportionate number, as non-Q wave myocardial infarction usually accounts for only $20-30 \%$ of all myocardial infarct ${ }^{27}$ ) - possibly reflecting a tendency to have more subendocardial ischaemia. Subendocardial ischaemia is thought to be exacerbated by adenosine mediated transmyocardial steal. ${ }^{26}$ Thus this group of patients could be self selected responders to SCS.

Indeed this group of patients seems to be unique-despite having severe coronary disease they are actually survivors, which ironically is one of the major problems. They have often survived multiple infarcts, but they still continue to have regular admissions and the attrition rate is low, as we have already seen. Hospital admissions are prolonged, as further treatment options are limited. The cost of SCS is relatively high (approximately $£ 6000$ ), but a bed on the coronary care unit in our hospital costs approximately $£ 850$ a day, and the general cardiology ward costs around $£ 200$ a day (table 4). The device undoubtedly improves the patient's symptoms, and it is likely 
that the reduction in the need for hospital admissions will cover the initial outlay for the device (within three years according to our estimates in table 4).

In summary, SCS is a safe, well tolerated, and effective treatment for refractory angina. It is relatively simple to employ, and has a perioperative mortality of zero, and little morbidity. The effects of this treatment are powerful enough to decrease the need for hospital admission without apparently masking acute coronary syndromes or producing excess mortality, even several years after implantation.

\section{LIMITATIONS OF THE STUDY}

This was a retrospective observational study and there is no placebo control group included. Patients have acted as their own controls by comparing admission rates before and after SCS in a non-randomised fashion. Thus we cannot rule out the possibility of placebo effect, although we feel the persistence of benefit with SCS (up to nine years in some patients) makes it unlikely that placebo response is the sole mechanism of action.

PE is funded by NHSE South and West R\&D Directorate.

1 Murphy DF, Giles KE. Dorsal column stimulation for pain relief from refractory angina pectoris. Pain 1987;28:365-8. 2 Mannheimer C, Augustinsson LE, Carlsson CA, et al. Epidural spinal electrical stimulation in severe angina pectoris. Br Heart F 1988:59:56-61.

3 Sanderson JE, Brooksby P, Waterhouse D, et al. Epidural spinal electrical stimulation for severe angina: a study of its effect on symptoms, exercise tolerance and degree of ischaemia. Eur Heart 7 1992;13:628-33.

4 Dejongste MJL, Hautvast RWM, Hillege JL, et al, on behalf of the working group on neurocardiology. Efficacy of spinal cord stimulation as adjuvant therapy for refractory angin pectoris. 7 Am Coll Cardiol 1994:23:1592-7.

5 Sanderson JE, Ibrahim B, Waterhouse D, et al. Spinal electrical stimulation for refractory angina-long term clinical outcome and safety. Eur Heart $\mathcal{F} 1994 ; 15: 810-14$.

6 Dejongste MJL, Haaksma J, Hautvaust RWM, et al. Effects of spinal cord stimulation on myocardial ischaemia during daily life in patients with severe coronary artery disease. A daily life in patients with severe coronary artery disease. A prospective ambulatory

7 Kujacic V, Eliasson T, Mannheimer C, et al. Assessment of spinal cord stimulation (SCS) on left ventricular function in patients with severe angina pectoris: an echocardiographic study. Eur Heart f 1993;14:1238-44.
8 Mannheimer C, Eliasson T, Andersson B, et al. Effects of spinal cord stimulation in angina pectoris induced by pacing and possible mechanisms of action. BMF 1993;307: $477-80$

9 Anderson C, Hole P, Okhoj H. Spinal cord stimulation as a pain treatment for angina pectoris. The Pain Clinic 1995;8: 333-9.

10 Anderson C, Hole P, Oxhoj H. Does pain relief with spinal cord stimulation for angina conceal myocardial infarction? Br Heart f 1994;71:419-21.

11 Collins PD, Nicholls BJ, Titley G, et al. Quality of life after spinal cord stimulation for angina pectoris. Proceedings of the Pain Society. Annual Scientific Meeting, April 1997.

12 Eliasson T, Augustinsson LE, Mannheimer C. Spinal cord stimulation in severe angina pectoris - presentation of current studies, indications and clinical experience. Pain 1996; 65:169-79.

13 Mulcahy D, Knight C, Stables R, et al. Lasers, burns, cuts, tingles and pumps: a consideration of alternative treatments for refractory angina. Br Heart $\mathcal{F}$ 1994;71:406-7.

14 Dooley D, Kasprak M. Modification of blood flow to the extremities by electrical stimulation of the nervous system. South Med f 1976;69:1309-11.

15 Meglio M, Cioni B, Dal Lago A, et al. Pain control and improvement of peripheral blood flow following epidural spinal cord stimulation: case report. $\mathcal{f}$ Neurosurg 1981;54: $821-3$.

16 Augustinsson LE, Carlsson CA, Holm J, et al. Epidural electrical stimulation in severe limb ischaemia. Pain relief, ncreased blood flow, and a possible limb-saving effect. Ann Surg 1985;202:104-10.

17 Fiume D. Spinal cord stimulation in peripheral vascular pain. Appl Neurophysiol 1983;46:290-4.

18 Tallis RC, Illis LS, Sedgwick EM, et al. Spinal cord stimulation in peripheral vascular disease. $f$ Neurol Neurosurg Psychiatry 1983;46:478-84.

19 Broseta J, Barbera J, de Vera JA, et al. Spinal cord stimulation in peripheral arterial disease. A co-operative study. F Neurosurg 1986;64:71-80.

20 Jacobs MJ, Jorning PJ, Joshi SR, et al. Epidural spinal cord electrical stimulation improves microvascular blood flow in severe limb ischaemia. Ann Surg 1988;207:170-83.

21 Hautvast RWM, Blanksma PK, DeJongste MJL, et al. Effect of spinal cord stimulation on regional myocardial blood flow assessed by positron emission tomography. Am f Cardiol 1996;77:462-7.

22 Crea F, Pupita G, Galassi A, et al. Effect of theophylline on myocardial ischaemia. Lancet 1989;i:683-6.

23 Gaspardone A, Crea F, Iamele $\mathrm{M}$, et al. Bamiphylline improves exercise-induced myocardial ischaemia through a novel mechanism of action. Circulation 1993;88:502-8.

24 Sanderson JE. Epidural neurostimulators for pain relief in angina. Br Heart $\mathcal{F}$ 1990;63:141-3.

25 Norsell H, Eliasson T, Mannheimer C, et al. Effects of pacing induced myocardial stress and spinal cord stimulation on whole body and cardiac norepinephrine spillover. Eur Heart F 1997;18:1890-6.

26 Ganz P, Braunwauld E. Coronary blood flow and myocardial ischaemia. In: Braunwauld $\mathrm{E}$, ed. Heart disease, 5 th ed. Philadelphia: WB Saunders, 1997:1168-74.

27 Swanton RH. Coronary artery disease. In: Swanton RH, ed: Cardiology, 3rd ed. Oxford: Blackwell Scientific Publications, 1994:207-8. 